TRANSLATION

\section{RAPping with ribosomes}

Ribosomes are large complexes comprising RNA and proteins, which are primarily dedicated to protein synthesis. Interestingly, mammalian ribosomes also associate with various accessory proteins, forming the ribosome-protein interactome. However, the composition and function of this 'ribo-interactome' is poorly understood.

To study the mammalian ribo-interactome, Barna and colleagues endogenously tagged selected ribosomal proteins in mouse embryonic stem cells, purified ribosomes from these cells, and analysed the ribosomal proteome by mass spectrometry. They narrowed their analysis to proteins that directly interacted with ribosomes (that is, those that did not bind to ribosomal RNA or the nascent polypeptide), and referred to these as ribosome-associated proteins (RAPs). They identified $\sim 430$ RAPs, which fall into three categories: proteins that are involved in mRNA modifications; enzymes that mediate post-translational modifications (PTMs); and proteins that are implicated in basic cellular functions, including the cell cycle, reduction-oxidation (redox) homeostasis, and metabolism.

One of the identified RAPs was

UFL1, which is an enzyme that mediates protein ufmylation - a metazoan-specific PTM that resembles ubiquitylation. Three ribosomal proteins and one translation initiation factor were found to be ufmylated, indicating that the association of UFL1 with ribosomes has functional importance.

The authors then focused on one of the metabolism-associated RAPs, pyruvate kinase muscle isozyme (PKM), which catalyzes the final step of glycolysis, mediating the transfer of a phosphoryl group from phosphoenolpyruvate to ADP to produce ATP. Artificially tethering PKM to ribosomes and measuring translation efficiency revealed that PKM is an activator of translation. PKM was shown to

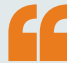

RAPs can bind

to a subset of ribosomes and regulate translation at defined subcellular localizations

5

interact with RNA, including different protein-coding mRNAs. Interestingly, a large proportion of the mRNAs that were directly bound by PKM encode endoplasmic reticulum (ER)-resident proteins, suggesting that PKM might promote translation of this subset of mRNAs. Indeed, PKM-containing ribosomes were associated with components of the ER protein translocon, and ER-bound ribosomes were enriched in PKM. Moreover, ER-translated mRNAs were reduced after PKM depletion.

This study suggests that specific RAPs can bind to a subset of ribosomes and regulate translation at defined subcellular localizations. It thus reveals that the mammalian ribo-interactome is highly complex and that RAPs may provide additional levels of translation regulation.

\section{Paulina Strzyz}

ORIGINAL ARTICLE Simsek, D. et al. The mammalian ribo-interactome reveals ribosome functional diversity and heterogeneity. Cell 169 , 1051-1065 (2017)

FURTHER READING Xue, S. \& Barna, M.

Specialized ribosomes: a new frontier in gene regulation and organismal biology. Nat. Rev. Mol. Cell Biol. 13, 355-369 (2012) 